\title{
Urgences
}

\section{Les ordures ménagères (l'art du coprotexte)}

\section{Ghislain Bourque}

Numéro 19, janvier 1988

Le tour du texte

URI : https://id.erudit.org/iderudit/025449ar

DOI : https://doi.org/10.7202/025449ar

Aller au sommaire du numéro

Éditeur(s)

Urgences

ISSN

0226-9554 (imprimé)

1927-3924 (numérique)

Découvrir la revue

Citer ce document

Bourque, G. (1988). Les ordures ménagères (l'art du coprotexte). Urgences, (19), 82-85. https://doi.org/10.7202/025449ar

Ce document est protégé par la loi sur le droit d'auteur. L’utilisation des services d’Érudit (y compris la reproduction) est assujettie à sa politique d'utilisation que vous pouvez consulter en ligne.

https://apropos.erudit.org/fr/usagers/politique-dutilisation/
Cet article est diffusé et préservé par Érudit.

Érudit est un consortium interuniversitaire sans but lucratif composé de l’Université de Montréal, l’Université Laval et l’Université du Québec à Montréal. Il a pour mission la promotion et la valorisation de la recherche. https://www.erudit.org/fr/ 


\section{Ghislain Bourque \\ LES ORDURES MÉNAGÈRES \\ (L'ART DU COPROTEXTE)}

De crainte que la PAILLE en TRESSE ne GRAVE son TRÉPAS, le mammifère rongeur demeure à distance respectable. Préférant s'occuper à des brouillons échappés d'une table où l'architecture est à l'honneur.

C'est un travail ingrat que celui de "rat de bibliothèque». Cela demande de l'adresse - surtout pour atteindre avant qu'ils ne se décomposent les restes détachés des cogitations de la clientèle régulière - de la ruse même - que l'on songe aux multiples pièges tendus au-dessus d'un détritus appétissant (comme celui par exemple de cette paille tressée d'une manière coulissante oscillant sur la crête d'une note personnelle parfumée, roulée en boule et s'expansionnant juste assez pour permettre l'échappement de quelques rimes - de la santé aussi, car si atteindre la nourriture relève souvent de l'épreuve initiatique, la consommer demeure un art. Tant on s'incommode parfois d'apprêter ce qu'une feuille donne en mille, ce que des mots offrent en tas de lettres.

Pour sa mise en fonction et son recyclage permanents, la bibliothèque a fait l'objet d'un caprice d'architecture. C'est un dispositif simple mais efficace où la bonne volonté et le civisme des lettrés comptent pour peu. Ils y adhèrent souvent malgré eux, plongés qu'ils sont dans des cogitations ou des exercices de style absorbants. Aussi ne réalisent-ils pas tout à fait ce qui, sous eux, à quelques pas de leurs pieds, se passe. En fait, ils n'ouvrent les yeux que le jour où, laissant de manière accidentelle tomber une pièce maitresse de leur travail (mécanisme fictionnel alambiqué, détail conceptuel élaboré, message personnel enflammé), ils se voient dans l'impossibilité de le récupérer. Puisqu'alors ils assistent impuissants à l'engloutissement de leur essentiel fragment, absorbé qu'il est par un plancher tenant lieu de grillage, où fentes, interstices et cavités accueillent sans retour tout reste échappé de leur table.

Dans les circonstances, on comprendra leur soudaine propension au drame. Celui imaginable quand vient définitivement se perdre sous vos yeux un document précieux. Et que vous ne pouvez le récupérer, sauf à y risquer la main, même si — véritable revers du savant mécanisme - souvent cela se résume à la seule perte d'un doigt: l'index surtout dont les mammifères vidangeurs raffolent. 
Alors, dans le silence besogneux de l'immense salle de travail, ça gesticule, ça crie, voire (cas extrêmement rare) ça perd connaissance. Cas rare et aussi litigieux, car si dans les deux premières circonstances les gardiens ont pour mission d'intervenir et de rapidement rétablir l'ordre, pour cette troisième rien n'y fait. Respectueux qu'ils sont de la consigne du non-recouvrement de tout ce qui va au plancher... Consigne héritée d'on ne sait qui - à supposer peut-être qu'elle fut l'objet d'une entente verbale avec le syndicat des rats de bibliothèque... Toujours est-il que pour ces quelques rares cas de perte de connaissance, il y a attroupement sous la grille. Savamment empilés, les rongeurs, comme dirigés par les tactiques de leur propre service de récupération, s'emploient à tirer une manche, ramener une main, mordiller une oreille... À cet effet, dans des circonstances que l'on peut qualifier de loufoques, on rapporte qu'une lectrice évanouie y a, parce que tombée face contre grillage, déjà laissé la plus large portion de son appendice nasal...! Ce à quoi auraient assisté les autres occupants de la bibliothèque dans une indifférence à peine camouflée.

Les jours de vaches maigres, qui toujours coïncident avec ces moments un peu magiques où le travail ne produit aucun reste, les rats s'impatientent. Si bien qu'au parcours dénudé de leur dépotoir, quelque frénésie s'installe, progressivement, jusqu'à ce qu'un débris, échappé on ne sait trop comment, vienne canaliser les marques de frustrations accumulées. Alors ce sont, entre rongeurs, des luttes féroces qui s'engagent. Luttes fratricides où l'esprit corporatif s'évapore, et au cours desquelles une note se déchire, un mot se fractionne jusqu'à, bien malgré lui, distribuer ses lettres à tout un chaçun.

Et, si rien n'y fait - nul brouillon, ni page détachée d'un dictionnaire un peu spécialisé, ni même quelque reste émietté d'un goûter absorbé avec discrétion --, nos vidangeurs, contenant leur agressivité, adoptent une autre stratégie.

Formant choeur, ils se mettent tous à couiner. Et ce d'une manière telle qu'en tout point multipliés les petits crịs viennent à progressivement moduler une fréquence où s'installe un insupportable sifflement. Ce qui a pour effet radical de distraire la clientèle et, consécutivement, de provoquer de menues erreurs de scription.

En l'absence de toùte observation un peu soutenue, on est porté à croire que les agissements des bandes de vidangeurs ne répondent à aucune hiérarchie particulière. Sur cette déduction singulière on se leurre. Nul travail dans cette fonction de récupération ne trahit un ordonnancement aussi spécifié. C'est à tout le moins ce que 
voudrait arriver à démontrer ce chercheur d'âge mûr, à la moustache fine et nerveuse, et dont les journées sont tout entières absorbées par les faits et gestes des rats.

Ainsi, de quelques observations initiées pratiquement au hasard, il s'est étonné d'abord que les rongeurs ne produisent aucun excrément visible (il le sait pour s'être appliqué à scruter au moyen d'une lunette d'approche le territoire par eux occupé); puis, qu'à leur mort, ils n'accusent nul cadavre en putréfaction (il faut dire qu'en ce cas l'utilisation de lunettes d'approche ne fut d'aucun secours, aussifut-elle remplacée par celle d'un multiplicateur de sensations olfactives... qui, au demeurant, ne donna rien de significatif).

Cela fút amplement suffisant pour déclencher en lui quelque appétit supplémentaire. Aussi, enhardi et devenu momentanément exalté par ce qu'il vit, s'est-il cru investi d'un pouvoir accru d'investigation. C'est d'ailleurs ainsi que, quadrillant le territoire déjà plafonné à claire-voie horizontale, il s'applique à enregistrer le détail des moindres comportements manifestés.

D'étónnement en étonnement, il note les agissements particuliers de ce qu'il nomme depuis son "étrange colonie». Notes qu'il garde jalousement, et dont la diffusion se limite à sa seule lecture. $\dot{A}$ l'exception toutefois de celle de quelques curieux qui, à plonger du regard par-dessus son épaule, arrivent à dérober un principe, deviner une contrainte, vérifier une hypothèse maintes fois reçue.

D'ailleurs, tout autour et comme malgré lui, des rumeurs circulent. De celles qui alimentent les réflexions les plus farfelues. Et que, comme malgré nous, l'on retient. À commencer par celle stipulant que les rats ne s'agitent pas devant tout reste de manière indifférenciée. Que bien au contraire ils semblent respecter un code dont l'éthique s'avère excessivement poussée.

Ainsi, pour un fragment échappé, seuls quelques-uns d'entre eux d'abord se manifestent, juchés sur leurs pattes de derrière, couinant de manière à indiquer où se trouve échue la marchandise. Désignée sous le vocable de «rôdeurs», cette catégorie de rongeurs limite son travail à signifier un lieu d'intervention: tout au plus! Laissant, une fois leur message enregistré, à une nouvelle escouade le loisir de répertorier la dépouille et d'en déterminer la répartition. Ces autres rats, que l'on nomme les «receleurs», accourent prestement sur les lieux de la perte et là, une fois le menu identifié, convoquent le ou les rongeurs appropriés. Car cette curiosité n'est pas des moindres: les rats ne s'alimentent ni auprès de toute victuaille, ni dans 
le désordre collectif. Mais bien plutôt, à ce qu'il semblerait, en répondant au repas selon leur catégorie.

C'est ce que confirme, une fois la table mise, la présence du "géniteur», dont le rôle consiste à d'abord analyser les restes hérités, et à ensuite tisser les liens qui, entre ces restes et quelques autres déjà recrutés, s'imposent. Ainsi seulement s'avère-t-il en mesure de gérer le mode de consommation qui en la circonstance s'impose.

Ce programme, où les comportements semblent répondre à un ordonnancement des plus stricts, n'est pas sans troubler la quiétude de la clientèle. Persuadée qu'elle est, quand bien même instruite de menues observations distraites, que le type de reste absorbé par le rongeur élu n'est pas sans conséquence sur son évolution propre. À cet effet d'ailleurs de sensibles modifications ont été observées. Remarquant chez les uns une réelle propension à se développer d'une manière fantastique, alors que chez d'autres c'est le comportement ou encyclopédique ou équationnel qui domine.

En ces cas, il convient de se rendre à l'évidence: toute absorption désignée entraîne, dans l'organisme même du vidangeur, une construction soignée de son code génétique. Laquelle construction pouvant à l'usage se traduire en mutations qui, du point de vue de la clientèle, pourraient s'avérer catastrophiques. C'est en partie d'ailleurs ce qui explique leur récente préoccupation à ne point se départir d'extraits trop textuellement marqués. Puisqu'au demeurant, qu'auraient-ils à gagner de ce qu'un rat, si affamé soit-il, se trouve indifféremment GAVÉ D'UN RESTE ÉPARS PILLÉ! 